\title{
STUDIES ON MYCOBACILLIN DERIVATIVES \\ IV. O-METHYLATION OF THE ANTIBIOTIC AND IDENTIFICATION OF THE ACTIVE TYROSINE RESIDUE
}

\author{
P. C. BANERJEE \\ Indian Institute of Experimental Medicine, Calcutta-700 032, India
}

(Received for publication June 6, 1977)

\begin{abstract}
Methylation of mycobacillin with dimethyl sulfate and methyl iodide produced respectively mono- and di-methyl derivatives. The products were inactive against fungal spores and erythrocytes. Tyrosine hydroxyl groups only were methylated; in the monomethyl derivative, the hydroxyl of the tyrosine residue in position 6 is methylated, whereas the hydroxyl of the tyrosine in position 4 remains free; both hydroxyl groups are methylated in the dimethyl derivative. The results indicate that the hydroxyl group of the tyrosine residue in position 6 is essential for the biological activity of mycobacillin.
\end{abstract}

Mycobacillin is a cyclic tridecapeptide ${ }^{1 \sim 3)}$ containing three hydroxyl groups, one in the serine residue and two in the tyrosine residues, and seven carboxyl groups in the glutamic and aspartic acid residues. Several derivatives of this antibiotic were prepared, and their antifungal, hemolytic and serum inactivation properties studied ${ }^{4}$ 7). It was observed that acetylation of the two tyrosyl hydroxyl groups renders the molecule nonhemolytic ${ }^{7)}$. Since a monoacetyl derivative could not be prepared it is not possible to state which of the tyrosyl hydroxyl groups is essential for hemolysis. Therefore, a mono- and a dimethyl tyrosine derivatives of the antibiotic were prepared and both of them were found to be biologically inactive. This paper describes the preparation, properties and characterization of these methyl derivatives and identification of the methylated tyrosine residue in the monomethyl derivative.

The specific position of methylation in the monomethyl derivative was determined taking advantage of the preferential hydrolysis of the molecule at the two $\gamma$-peptide bonds under mild conditions ${ }^{3)}$ (Scheme 1). Dinitrophenylation of the peptide containing the two tyrosine residues obtained from this hydrolysis, and subsequent acid hydrolysis of the DNP-peptide would give either one or two different DNP-amino acids depending upon the site of methylation in the original antibiotic. From the 4-O-methyl mycobacillin a mixture of two DNP- derivatives (DNP-tyrosine- $\mathrm{OCH}_{3}$ and O-DNPtyrosine) would be obtained, whereas the 6-O-methyl product would yield only one DNP-derivative (di-DNP-tyrosine). These DNP-derivatives may be easily characterized indicating the specific tyrosine residue methylated.

\section{Materials and Methods}

Mycobacillin was extracted from the culture filtrate of Bacillus subtilis $\mathrm{B}_{3}$ and was purified. ${ }^{8}$ ) Standard O-DNP and di-DNP derivatives of tyrosine were prepared according to SANGER ${ }^{9}$, and LEVY and CHUNG ${ }^{10)}$ respectively. 1-Fluoro-2,4-dinitrobenzene (FDNB) was purchased from E. Merck (Germany). All the organic solvents used were of analytical grade.

Methylation of mycobacillin 
Scheme 1. Probable DNP-amino acids from mono-methyl mycobacillin.

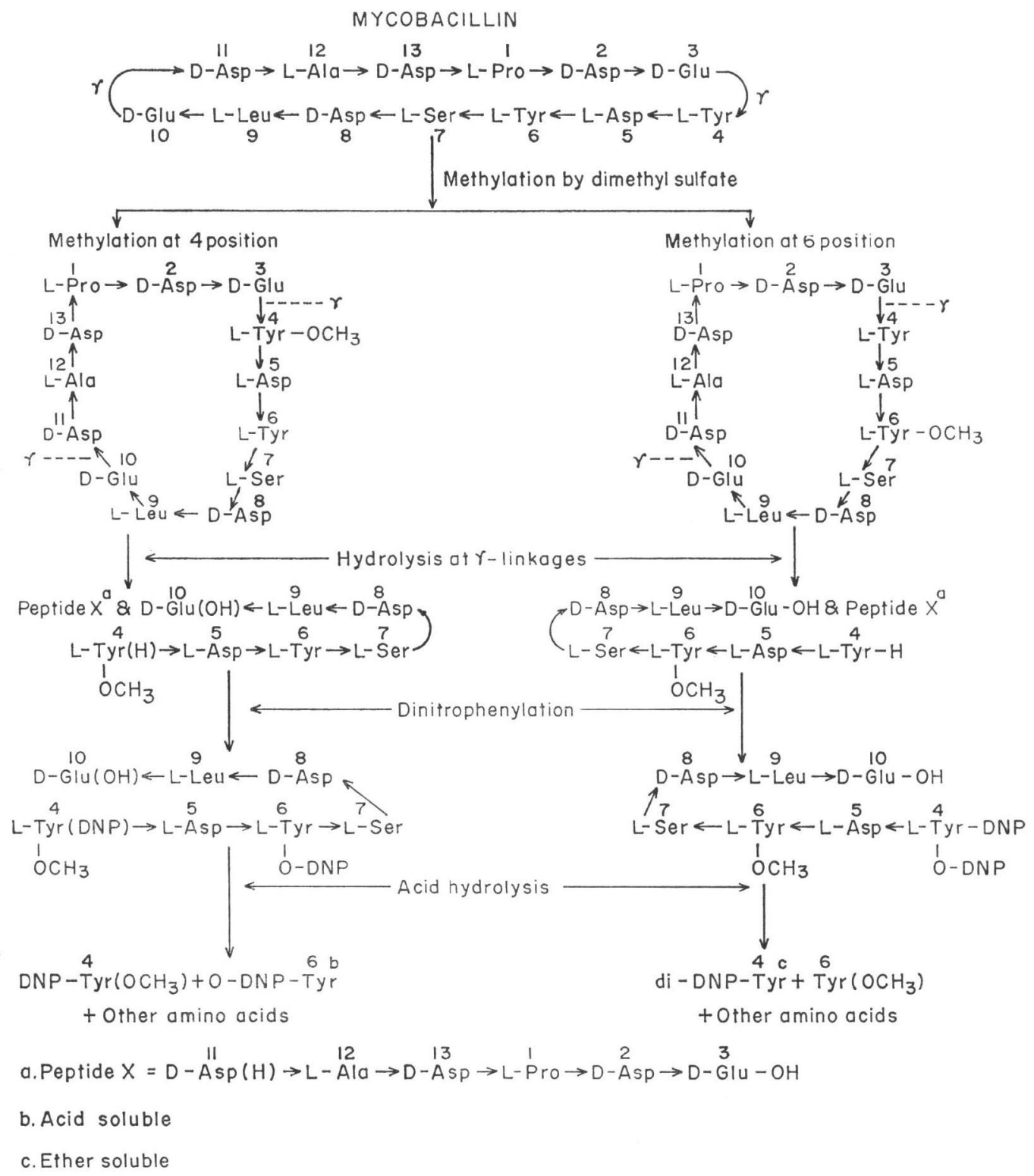

Mycobacillin was methylated with dimethyl sulfate and methyl iodide following SARGES and WITKOP $^{11)}$, and JAMES and SYNGE ${ }^{12)}$ respectively.

1. Methylation with dimethyl sulfate: Mycobacillin (100 mg) was solubilized in $50 \mathrm{ml}$ of dimethyl sulfoxide - dimethylformamide $(1: 1, \mathrm{v} / \mathrm{v})$ with a few drops of water. Barium hydroxide $(1.5 \mathrm{~g})$ and dimethyl sulfate $(2.0 \mathrm{ml})$ were added and the mixture was stirred for 5 hours at room temperature. Two $\mathrm{ml}$ of $8 \mathrm{~N}$ ammonium hydroxide were then added and stirring was continued for another 30 minutes. The mixture was then diluted with water $(200 \mathrm{ml})$ and chloroform $(250 \mathrm{ml})$, and shaken for 5 minutes. The chloroform layer was then separated, washed with water $(5 \times 125 \mathrm{ml})$, dehydrated over magnesium sulfate and evaporated to a small volume. The derivative was precipitated 
from this concentrated solution with dry ether, centrifuged and crystallized from methanol. The product (derivative I) was found to be chromatographically pure.

2. Methylation with methyl iodide: Mycobacillin (100 mg) was dissolved in $20 \mathrm{ml}$ of dioxan and a minimum volume of water. Methyl iodide $(4 \mathrm{ml})$ and freshly prepared moist silver oxide $(2.0 \mathrm{~g})$ were then added and the reaction was allowed to take place for 30 minutes at $60^{\circ} \mathrm{C}$. After filtration of the reaction mixture, the filtrate was concentrated and evaporated in vacuo. The residue was extracted with chloroform to separate unreacted mycobacillin from the product and the extract centrifuged. The supernatant was evaporated to dryness and the derivative crystallized from the same solvent. The product (derivative II) was homogeneous as evidenced by thin-layer chromatography.

Estimation of free hydroxyl groups

The number of free hydroxyl groups in the O-methyl derivatives was determined by acetylating the compounds with acetic anhydride and pyridine, and estimating colorimetrically the number of acetyl groups ${ }^{4)}$. The values indirectly give the extent of hydroxyl methylation in the derivatives.

Estimation of serine in the derivatives

The serine residue of mycobacillin may remain unchanged if methylation of the antibiotic is incomplete. Hence, the relative amount of this amino acid in mycobacillin and its derivatives was determined. The compounds were hydrolyzed with acid, and the aspartic and glutamic residues removed from the neutralized hydrolyzates by passing through an acid alumina column ${ }^{13}$. Serine was then separated from other amino acids by chromatography on a Dowex $50 \mathrm{~W}-\mathrm{X} 8\left(\mathrm{H}^{+}\right)$column using $1 \mathrm{~N} \mathrm{HCl}$ as eluent ${ }^{14}$ ), and estimated by the periodate-formaldehyde procedure ${ }^{15}$.

Hydrolysis of mycobacillin derivatives at $\gamma$-linkages and separation of the resulting peptides

The method of SENGUPTA et al..$^{3)}$ was followed to hydrolyze mycobacillin derivatives at $\gamma$-peptide bonds and to separate the two newly formed peptides. Only the tyrosine (or its derivative)-containing peptides were further processed after their elution from the paper-chromatograms.

Dinitrophenylation of the peptides

Dinitrophenylation of the eluted peptides was carried out following SANGER's method ${ }^{9}$, as modified by LOCKHART and ABRAHAM ${ }^{16)}$.

Hydrolysis of DNP-peptides and separation of acid and ether soluble DNP-amino acids

Dinitrophenylated peptides were dissolved in $0.5 \mathrm{ml}$ of $6 \mathrm{~N} \mathrm{HCl}$ and hydrolyzed at $105^{\circ} \mathrm{C}$ for 8 hours in evacuated sealed tubes. $\mathrm{HCl}$ was then completely removed over $\mathrm{NaOH}$ pellets in vacuo $; 0.5 \mathrm{ml}$ of $1 \mathrm{~N} \mathrm{HCl}$ was added to the dried material and the solution extracted thrice with $0.5-\mathrm{ml}$ portions of ether. The combined ether extract was washed twice with a small volume of $0.1 \mathrm{~N} \mathrm{HCl}$. Ether and water extracts were evaporated to dryness and the residues examined for DNP-amino acids.

Thin-layer chromatography of DNP-amino $\operatorname{acids}^{17}$ )

Thin-layer chromatography was done on silica gel $\mathrm{G}$ using the solvent systems a) n-propanol $34 \% \mathrm{NH}_{4} \mathrm{OH}(70: 30, \mathrm{v} / \mathrm{v})$, and $\left.\mathrm{b}\right)$ chloroform - benzyl alcohol - acetic acid (70:30:3, v/v), for the water-soluble and the ether-soluble fractions respectively. Solvent system c), consisting of $n$-butanol saturated with $28 \% \mathrm{NH}_{4} \mathrm{OH}$, was also used for both fractions. DNP-amino acids were visualized under ultraviolet radiation.

Determination of antifungal and hemolytic activities

Antifungal and hemolytic activities of the derivatives were determined as described previously $y^{3,6}$.

\section{Results}

\section{Properties of the Derivatives}

The O-methyl derivatives were chromatographically homogeneous (Table 1). Unlike mycobacillin they are readily soluble in chloroform, but insoluble in dilute acid or alkali. Derivative I gives colour with diazotized sulfanilic acid and has an UV-absorption maximum similar to that of the parent antibiotic, whereas derivative II lacks these properties, indicating the presence of a free 
Table 1. Rf values, and methoxyl and serine content of mycobacillin derivatives

\begin{tabular}{|c|c|c|c|c|c|c|}
\hline \multirow{2}{*}{ Compound } & \multicolumn{2}{|c|}{ Rf values ${ }^{a)}$} & \multirow{2}{*}{$\begin{array}{c}\text { Moles of free } \\
\text { hydroxyl } \\
\text { group per } \\
\text { mole }^{\mathrm{d})}(\mathrm{x})\end{array}$} & \multirow{2}{*}{$\begin{array}{c}\text { Moles of O- } \\
\text { methyl group } \\
\text { per mole } \\
(3-x)\end{array}$} & \multirow{2}{*}{$\begin{array}{l}\text { Moles of } \\
\text { serine per } \\
\text { mole }^{\mathrm{e})}(\mathrm{y})\end{array}$} & \multirow{2}{*}{$\begin{array}{c}\text { Relative } \\
\text { molar value } \\
\text { of serine }\end{array}$} \\
\hline & Solvent $\mathrm{A}^{\mathrm{b})}$ & Solvent $\mathrm{B}^{\mathrm{c})}$ & & & & \\
\hline Derivative I & 0.65 & 0.52 & 1.82 & 1.18 & 0.82 & 1.12 \\
\hline Derivative II & 0.90 & 0.82 & 1.05 & 1.95 & 0.68 & 0.93 \\
\hline Mycobacillin & 0.02 & 0.05 & 3.10 & nil & 0.73 & 1.00 \\
\hline
\end{tabular}

a) Silica gel $\mathrm{G}$ plates were used.

b) Solvent system A: Chloroform - methanol (6:1, v/v).

c) Solvent system B: $n$-Butanol - water - acetic anhydride (23:1:1, v/v).

d) Estimated after acetylation of free groups.

e) Correction for 0-hour hydrolysis was not made.

phenolic group in derivative I, but not in derivative II.

Extent of Methylation

Moles of methoxyl groups per mole of derivatives I and II were found to be 1.18 and 1.95 respectively, the values being obtained indirectly from the molar proportion of acetyl groups in the acetylated derivatives (Table 1). Thus, of the three hydroxyl groups of mycobacillin two remain free in derivative I and one in derivative II.

\section{Serine Content in the Derivatives}

Table 1 shows the relative molar values of serine to be 1.12 and 0.93 in derivative I and II respectively, taking the value for mycobacillin as 1 . Thus, the serine hydroxyl group is free in both methylated derivatives, indicating that of the two tyrosine residues of mycobacillin one has been methylated in derivative I and both in derivative II.

Biological Activity of the Derivatives

Derivative I, with one methylated tyrosine residue in the molecule, is inactive against Aspergillus niger spores and erythrocytes even at the concentration of $200 \mu \mathrm{g} / \mathrm{ml}$ tested; derivative II has similar property. Hence, it appears that only one tyrosine residue of mycobacillin is primarily responsible for its biological activity.

\section{Characterization of DNP-Amino Acids in the Hydrolyzate}

1. DNP-amino acids in the acid-soluble fractions.

Mycobacillin yielded one DNP-derivative in this fraction identified as O-DNP-tyrosine (Fig. 1A). But neither derivative I nor derivative II gave any acid-soluble DNP-amino acids. This indicated that the tyrosine residue in position 6 was methylated in both derivatives (Scheme 1).

2. DNP-amino acid in the ether-soluble fractions.

The same di-DNP-tyrosine was obtained in the ether-soluble fractions from derivative I and mycobacillin, whereas derivative II gave a different DNP-compound (Fig. 1B). This is consistent with the presence of a free tyrosine residue in position 4 of derivative $\mathrm{I}$.

\section{Discussion}

The present work is an attempt to determine whether one or both the hydroxyl groups of the tyrosine moieties of mycobacillin are essential for its biological activity. It was reported previously that acetylation of the tyrosyl hydroxyl groups of mycobacillin lowers its antifungal activity and destroys the hemolytic property ${ }^{4,7)}$. The results suggested that the tyrosine residues are essential for 
the hemolytic activity and that there is a difference in the mode of action of the antibiotic towards erythrocytes and fungi ${ }^{7}$. In the present study it was found that methylation of the tyrosine residue in position 6 is sufficient to destroy both the hemolytic and antifungal properties of mycobacillin, and as such a free phenolic hydroxyl group at position 6 is essential for the biological activity of the antibiotic.

It is of interest that acetylation of the tyrosine residue in position 6 in the diacetyl derivative of mycobacillin does not destroy, but lowers the antifungal activity ${ }^{4}$. Thus it appears that a few modifications like acetylation replacing this essential phenolic hydroxyl group of mycobacillin may partially retain the biological function of the group.

The results may be further argued in the context of our previous observations that esterification or reduction of carboxyl groups of mycobacillin also causes partial or complete inhibition of its activity ${ }^{5} \sim 7$ ). On the basis of the present study, it may be suggested that esterification and especially reduction of the ionizable carboxyl groups may somehow make the target site inaccessible to the essential tyrosine hydroxyl group thus rendering the derivatives biologically inactive.

\section{Acknowledgement}

I am indebted to Dr. P. K. Dutra for his kind help.

\section{References}

1) Majumder, S. K. \& S. K. Bose: Mycobacillin, a new antifungal antibiotic produced by $B$. subtilis. Nature 181: 134 135, 1958

2) BANerJeE, A. B. \& S. K. Bose: Amino acid configuration of mycobacillin. Nature 200: 471, 1963

3) Sengupta, S.; A. B. BAnerJeE \& S. K. Bose: $\gamma$-Glutamyl and D- or L-peptide linkages in mycobacillin. Biochem. J. 121: 839 846, 1971

4) BanerjeE, P. C. \& S. K. Bose: Studies on mycobacillin derivatives. I. Acetyl derivatives of mycobacillin. J. Antibiotics 26: 257 260, 1973

5) Banerjee, P. C. \& S. K. Bose: Studies on mycobacillin derivatives. II. Ester derivatives of mycobacillin. Ind. J. Biochem. Biophys. 10: 302 304, 1973

6) Banerjee, P. C.; P. K. Dutta \& S. Sengupta: Studies on mycobacillin derivatives. III. Reduction of carboxyl groups of the antibiotic. J. Antibiotics 30: 420 422, 1977

7) BANeRJeE, P. C.: Lytic effect of mycobacillin and its derivatives on erythrocytes. Antimicr. Agents \& Chemoth. 12: 124 125, 1977

8) MAJUmder, S. K. \& S. K. Bose: Isolation and homogeneity of mycobacillin. Arch. Biochem. Biophys. 90: $154 \sim 158,1960$

9) SANGeR, F.: The free amino groups of insulin. Biochem. J. 39: 507 515, 1945

10) Levy, A. L. \& D. Chung: A simplified procedure for the synthesis of 2,4-dinitrophenyl (DNP)-amino acids. J. Am. Chem. Soc. 77: 2899 2900, 1955

11) Sarges, R. \& B. Witkop: Gramicidin A. V. The structure of valine and isoleucine gramicidin A. J. Am. Chem. Soc. 87: 2011 2020, 1965

12) JAmes, A. T. \& R. L. M. SYNGE: Non-peptide linkages in gramicidin. Biochem. J. 50: 109 118, 1951 
13) Kuhn, R. \& T. Wieland: Cited in Methods in Enzymology. III: pp. 575 578, by S. P. Bessman. Edited by S. P. Colowick \& N. O. Kaplan; Academic Press, Inc., New York, 1957

14) Chibnall, A. C.; C. Haselbach, J. L. Mangan \& R. W. Rees: Studies on the amide and C-terminal residues in proteins. 5. Estimation of asparagine and glutamine residues. Biochem. J. 68: 122 128, 1958

15) ReEs, M. W.: Studies on the amide and C-terminal residues in proteins. 4. Separation and quantitative determination of $\beta$-amino alcohols. Biochem. J. $68: 118 \sim 122,1958$

16) Lockhart, I. M. \& E. P. Abraham: The amino acid sequence in bacitracin A. Biochem. J. 58: 633 647, 1954

17) Brenner, M.; A. Niederwieser \& G. Pataki: Cited in Thin-layer Chromatography. pp. 730 786, Edited by E. Stahl, George Allen \& Unwin Ltd., London, Springer-Verlag, Berlin, Heidelberg, New York, 1969 